\title{
NUEVO SLING MASCULINO "ARGUS" PARA EL TRATAMIENTO DE LA INCONTINENCIA URINARIA DE ESFUERZO.
}

\author{
Jesús Moreno Sierra, Salomón Victor Romano', Isabel Galante Romo, Jerónimo Barrera \\ Ortega², Jesús Salinas Casado y Angel Silmi Moyano.
}

Servicio de Urologia. Hospital Clínico San Carlos. Universidad Complutense. Madrid.

Hospital Durand'. Buenos Aires. Argentina.

Servicio de Diagnóstico por la Imagen². Hospital Clínico San Carlos. Madrid. España.

\begin{abstract}
Resumen.- OBJETIVOS: La incontinencia urinaria masculina de esfuerzo, habitualmente se produce como consecuencia o secuela de una intervención quirúrgica prostática (prostatectomia radical abierta o laparoscópica, cirugía de la hiperplasia prostática benigna y esclerosis del cuello vesical: adenomectomia, resección transuretral convencional y/o bipolar, laser, etc) (1). Este tipo de cirugías puede tener efectos no deseados en la calidad de vida y las espectativas de los pacientes, aunque debemos ser conscientes que en los pacientes con adenocarcinoma de próstata el objetivo prioritario será curar el cáncer y en pacientes con síntomas obstructivos mejorar su calidad miccional. En la última década las intervenciones de compresión del bulbo uretral con cabestrillos han sido utilizadas con éxito en el tratamiento de la incontinencia urinaria de esfuerzo masculina (2), considerándose
\end{abstract}

Jesús Moreno Sierra

$\frac{0}{0}$
$\frac{1}{0}$
$\frac{1}{0}$
$\frac{1}{0}$
$\frac{1}{0}$
0
$\frac{1}{0}$
0

Servicio de Urología

Hospital Universitario San Carlos

Martín Lagos, s/n

28043 Madrid (España)

dr_jmoreno@hotmail.com

Trabajo recibido: 22 de mayo 2006. por grupos de gran experiencia en cabestrillos de gran efectividad en el tratamiento de la incontinencia urinaria post-prostatectomía a largo plazo (3).

Describir los elementos que componen el sistema Argus, sus indicaciones y la técnica quirúrgica mediante la que se implanta y ajusta adecuadamente, modificada de Schäeffer (2) y llevada a cabo por Victor Romano (1).

MÉTODO: Sistema Argus. El Sling está formado por tres componentes: sistema almohadillado radioopaco de espuma de silicona, impermeable a los fluidos corporales de $42 \mathrm{~mm} \times 26 \mathrm{~mm} \times 9 \mathrm{~mm}$, dos columnas de silicona formadas por multiples formaciones conicas, que permiten el reajuste del sistema y dos arandelas de silicona radioopacas $115 \mathrm{~mm}$ de diámetro y 2'9 mm de espesor) que pemiten una correcta fijación y reajuste (Figura 1). Una vez abierto el sistema, se recomienda sumergir el Sling en una solución antibiótica hasta su utilización.

CONCLUSIONES:

1. Se trata de un sistema seguro, fácil de implantar, reproducible, con pocas complicaciones y una buena relación coste beneficio.

2. Sus resultados son comparables al estandar de oro, pero con las siguientes ventajas: inmediata recuperación del control miccional y no requiere entrenamiento por parte del paciente.

3. Este trabajo no pretende aportar nuestra corta experiencia, que simplemente incluye 5 pacientes, aunque si queremos mencionar que se encuentran en la actualidad continentes y con una buena calidad de vida.

4. Nuestro objetivo será publicar nuestros resultados, cuando podamos aportar un seguimiento mínimo.

Palabras clave: Incontinencia urinaria de esfuerzo masculina. Cabestrillo regulable. Cabestrillo bulbouretral. Sistema Argus. 
Summary.- OBJECTIVES: Male stress urinary incontinence is usually a consequence or sequel of a prostatic surgical procedure /radical prostatectomy, surgery for BPH or bladder neck sclerosis: adenomectomy, conventional and/or bipolar transurethral resection, laser...). This kind of surgery may have undesirable effects on the quality of life and patients 'expectations, although we should bear in mind that the primary objective in patients with prostate adenocarcinoma is to cure cancer and for patients with obstructive lower urinary tract symptoms to improve their voiding quality.

Over the last decade, surgical procedures to compress the bulbar urethra with slings have been employed successfully in the treatment of male stress urinary incontinence, being considered highly effective in the treatment of post-prostatectomy incontinence in the long-term by groups with large experience.

To describe the elements of the Argus system, its indications, and the surgical technique for its implant and adjustment, modified from Schäeffer and carried out by Victor Romano.

METHODS: Argus system: The sling has three components: radiopaque cushioned system with silicone foam, 42 $\mathrm{mm} \times 26 \mathrm{~mm} \times 9 \mathrm{~mm}$, which is waterproof to body fluids; two silicone columns formed by multiple conical elements, which allow system readjustment; and two radiopaque silicone washers $115 \mathrm{~mm}$ diameter and 2.9 $\mathrm{mm}$ width) which enable proper fixation and readjustment (Figure 1).

Once the system is open, it is recommended to place the sling within antibiotic solution until implantation.

\section{CONCLUSIONS:}

1. It is a safe, easy to implant, reproducible system, with few complications and a good cost-benefit relation.

2. Results are comparable to the gold standard, but it has the following advantages: immediate voiding control recovery and no need for patient training.

3. This article does not intend to show our short experience with only five cases, but we want to mention that all of them are continent with a good quality of life.

4. Our objective will be to publish our results when we can show a minimal follow-up.

Keywords: Male stress urinary incontinence. Adjustable sling. Bulbourethral sling. Argus system.

\section{INTRODUCCIÓN}

La incontinencia urinaria masculina de esfuerzo, habitualmente se produce como consecuencia - secuela de una intervención quirúrgica prostática (prostatectomia radical abierta o laparoscópica, cirugía de la hiperplasia prostática benigna y esclerosis del cuello vesical: adenomectomia, resección transuretral convencional y/o bipolar, laser, etc) (1). Este tipo de cirugías puede tener efectos no deseados en la calidad de vida y las espectativas de los pacientes, aunque debemos ser conscientes que en los pacientes con adenocarcinoma de próstata el objetivo prioritario será curar el cáncer y en pacientes con síntomas obstructivos mejorar su calidad miccional.

De cualquier manera y con relativa frecuencia, estas intervenciones pueden tener efectos devastadores en la calidad de vida de los pacientes (1), sobre todo cuando les queda como secuela una incontinencia urinaria, que podrá deberse a tres causas fundamentales: alta presión, daño esfinteriano y/ o causas mixtas (1).

En la última década las intervenciones de compresión del bulbo uretral con cabestrillos han sido utilizadas con éxito en el tratamiento de la incontinencia urinaria de esfuerzo masculina (2), considerándose por grupos de gran experiencia en cabestrillos de gran efectividad en el tratamiento de la incontinencia urinaria post-prostatectomía a largo plazo (3).

En lo referente a la técnica que vamos a describir en este artículo debemos recordar sus origenes en Marshall y posteriormente en Schäeffer (2-6) por establecer las bases del tratamiento con sling, aunque fue Romano (1) quien modificó la técnica que hoy presentamos. Por supuesto no debemos olvidarnos de otros multiples antecedentes relacionados con los cabestrillos bulbouretrales, desde Kaufman y Puigvert, pasando por Raz, Franco y nuestro compatriota Sousa (7).

\section{OBJETIVO}

Describir los elementos que componen el sistema Argus, sus indicaciones y la técnica quirúrgica mediante la que se implanta y ajusta adecuadamente, modificada de Schäeffer (2) y llevada a cabo por Victor Romano (1).

\section{MATERIAL Y MÉTODO}

\section{Indicaciones}

Este cabestrillo está indicado en pacientes con incontinencia urinaria de esfuerzo, mantenida durante un periodo superior a 12 meses, secundaria a cirugía prostática (prostatectomia radical abierta 
- laparoscopica, cistoprostatovesiculectomía radical con neovejiga, cirugía de la hiperplasia prostática benigna, con cuello abierto y refractarios a manejo conservador) o por incompetencia esfinteriana de origen neurogénico. Está contraindicado cuando existe esclerosis de cuello, esclerosis de la anastomosis uretrocervical $y / o$ estenosis uretral, aunque en realidad son relativas siempre y cuando se traten y estabilicen las lesiones mencionadas como contraindicaciónes.

\section{Protocolo de estudio}

El diagnóstico se realiza con una adecuada historia y exploración física y se completa con la realización de las siguientes pruebas complementarias: uretrocistografía y estudio urodinámico y uretrocistoscopia.

Previo a la intervención quirúrgica el paciente deberá ser informado sobre las características del procedimiento y el sistema a implantar, con sus ventajas y posibles inconvenientes, riesgos y alternativas a este sistema. Este consentimiento que inicialmente sera verbal, deberá ser firmado por el propio paciente.

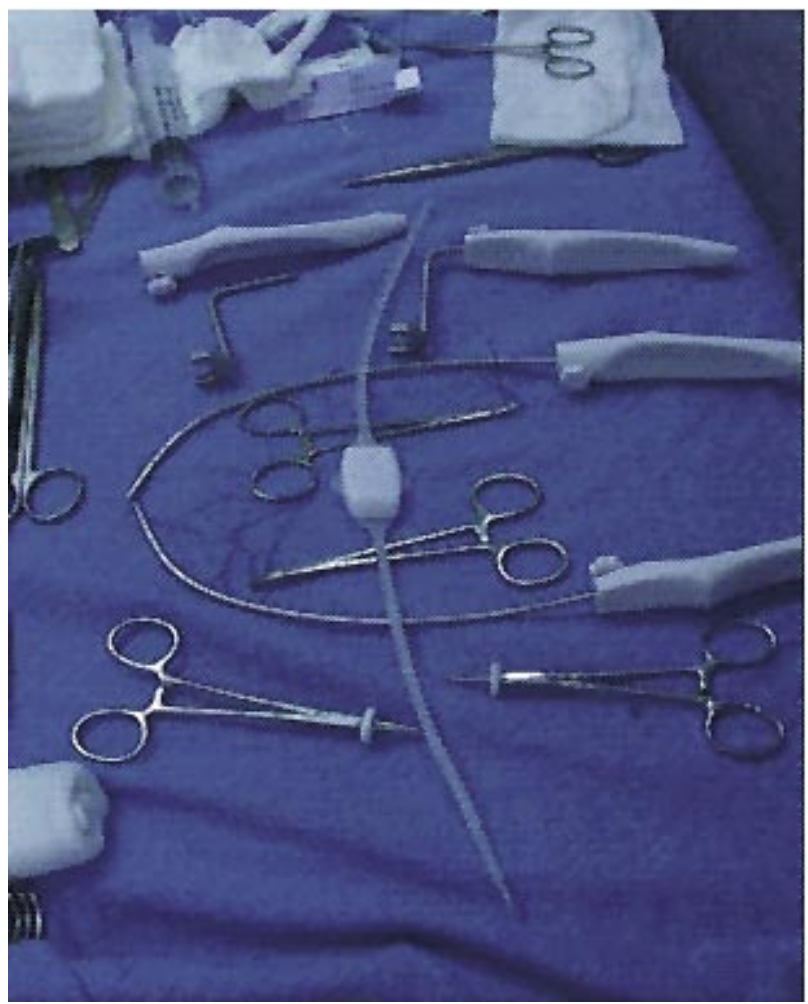

FIGURA 1. Sistema Argus. Almohadilla de espuma de siliconas, adosada a 2 columnas de conos de silicona, 2 arandelas (montadas en los clamps), las agujas de $90^{\circ}$ de mangos intercambiables, y el "posicionador"

\section{Sistema Argus}

El Sling está formado por tres componentes: sistema almohadillado radioopaco de espuma de silicona, impermeable a los fluidos corporales de 42 $\mathrm{mm} \times 26 \mathrm{~mm} \times 9 \mathrm{~mm}$, dos columnas de silicona formadas por multiples formaciones conicas, que permiten el reajuste del sistema y dos arandelas de silicona radioopacas $115 \mathrm{~mm}$ de diámetro y $2^{\prime} 9 \mathrm{~mm}$ de espesor) que pemiten una correcta fijación y reajuste (Figura 1).
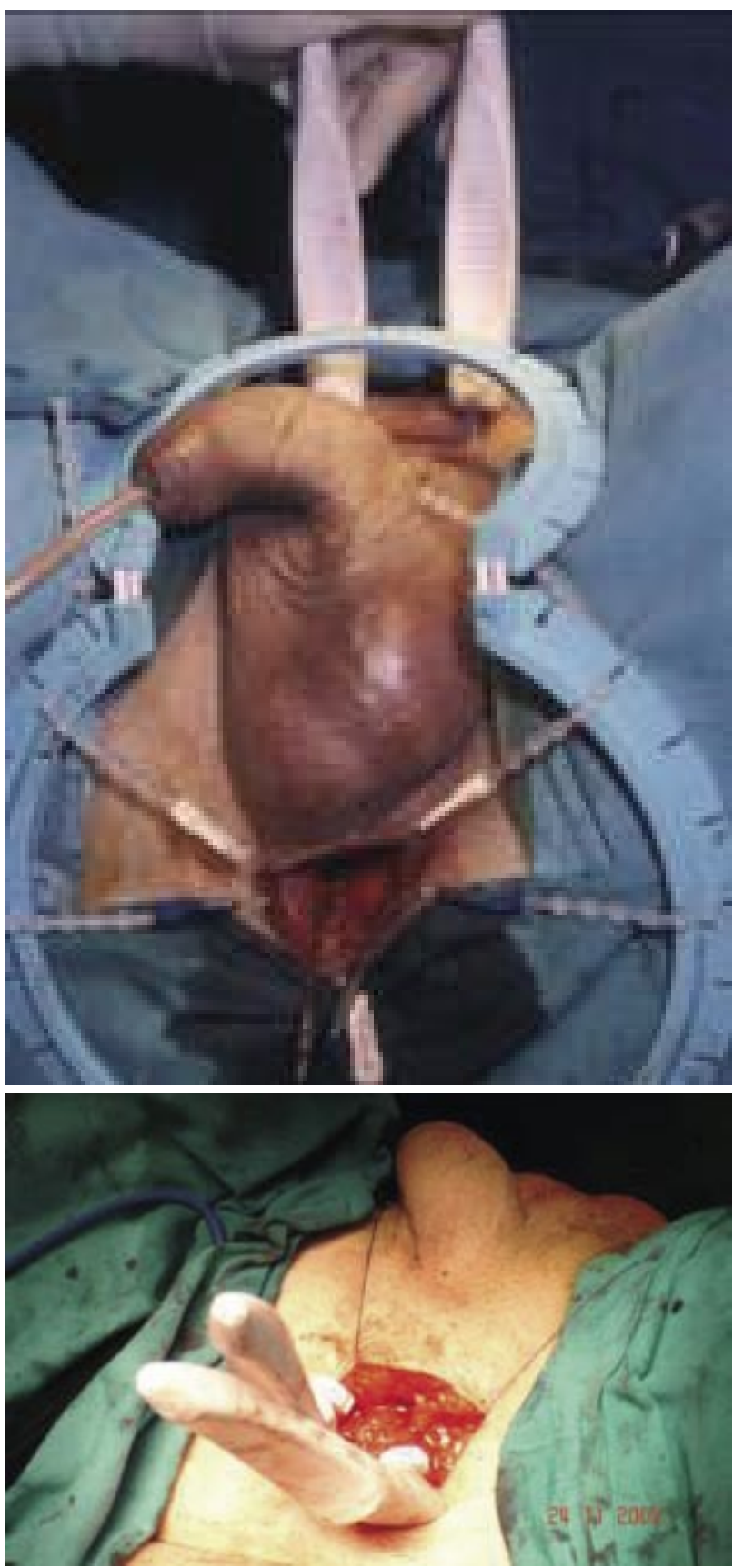

FIGURA 2. Cambio de posición de los mangos de las agujas. 


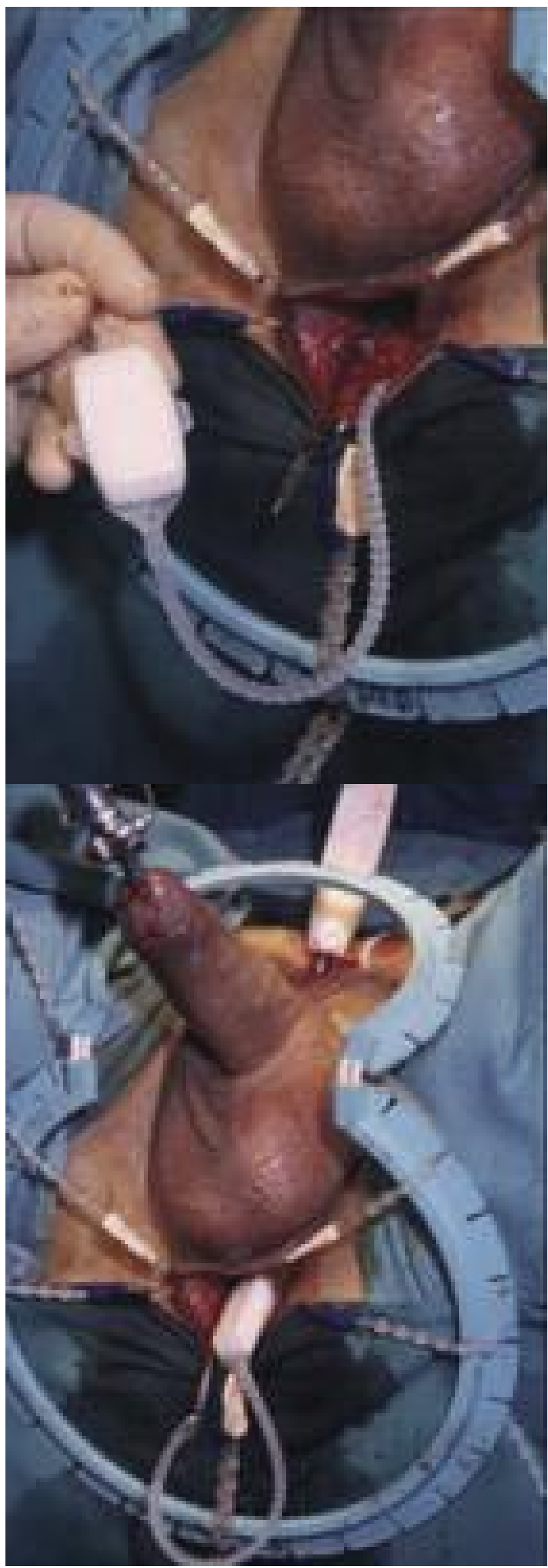

Una vez abierto el sistema, se recomienda sumergir el Sling en una solución antibiótica hasta su utilización.

\section{Material necesario}

Para implantar el sistema Argus se precisan dos agujas de acero inoxidable con mangos intercambiables y un posicionador (Figura 1). Previo a la intervención se aconseja insertar las agujas en los mangos plásticos correspondientes, haciendo coincidir las flechas marcadas en ambas piezas, así como ajustar el tornillo firmemente, tanto como sea necesario para impedir que la aguja se mueva.

\section{Técnica}

\section{Anestesia}

El procedimiento puede realizarse bajo anestesia raquídea o general.

\section{Preparación del paciente}

Limpieza mecánica del recto (enemas), profilaxis antibiotica, higiene y aseptización del área hipograstrica y urogenital con povidona yodada, sondaje uretrovesical (Foley $n^{\circ}$ 18), colocación del paciente en posición adecuada (litotomia y moderado Trendelemburg) y colocación del campo quirúrgico.

\section{Técnica quirúrgica paso a paso}

- Acceso suprapúbico.

Se realiza una incisión suprapúbica de unos $4-6$ cms, profundizando en el tejido celular subcutáneo hasta visualizar la aponeurosis, que deberá respetarse. La hemostasia deberá ser cuidadosa, con la finalidad de disminuir el riesgo de infección local. Opcional-

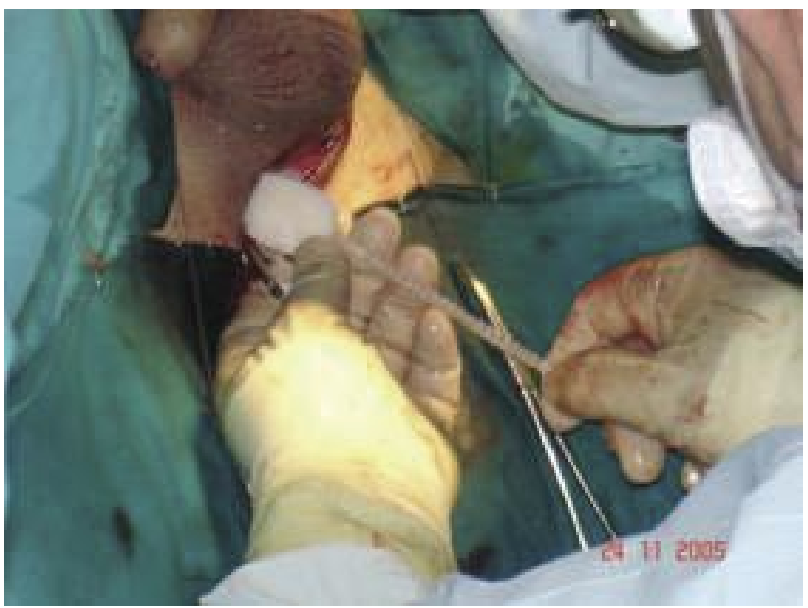

FIGURA 3. Enhebrado y paso de columnas. 

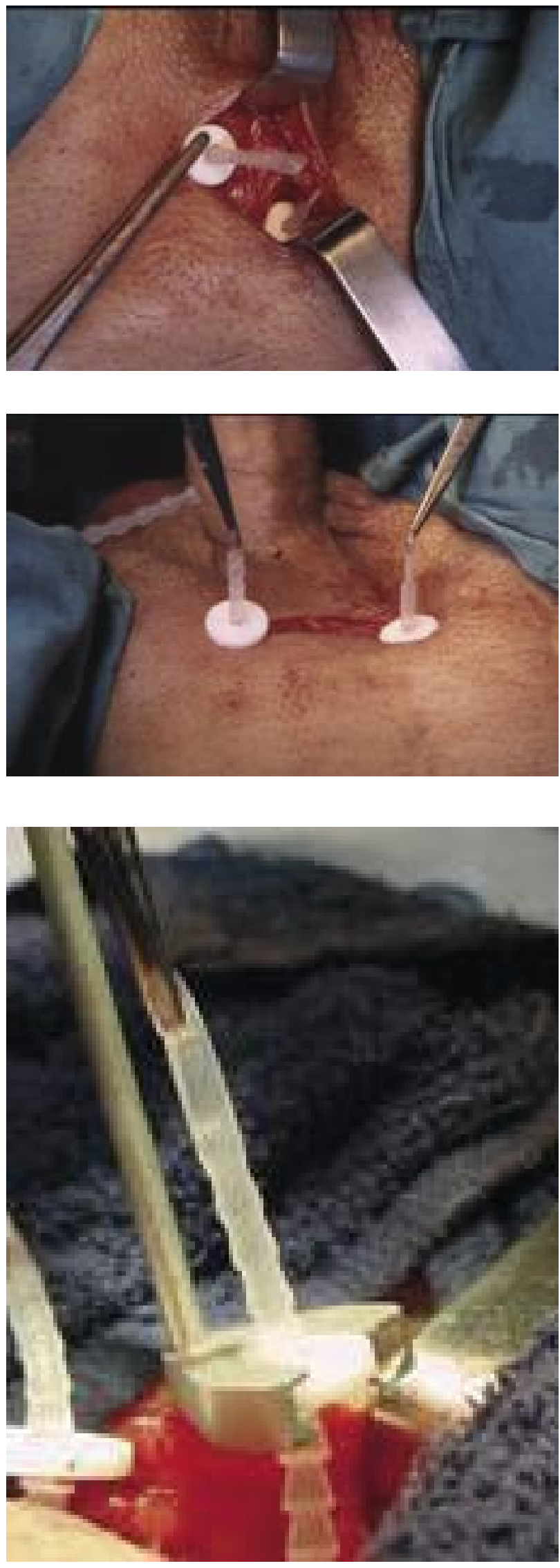

FIGURA 4. Colocación de las arandelas. Posicionador (imagen inferior). mente pueden ser confeccionadas 2 incisiones de 2-3 cms cada una de ellas.

- Acceso perineal. Consta de los siguientes pasos: I. Incisión perineal media de unos 5-7 cms.

II. Disección del espacio triangular localizado entre la uretra bulbar y la rama isquiopubiana la la que se adhiere la raíz del cuerpo cavernoso o crura a ambos lados), hasta alcanzar el suelo del espacio triangular formado por la capa superficial de la aponeurosis perineal. Así se expone el músculo bulbocavernoso y lateralmente las ramas isquiopubianas.

III. Paso de agujas a ambos lados de la uretra bulbar, desplazando y protegiendo la uretra con un dedo de la mano contraria a la que manipula la aguja de punción, en dirección hacia la incisión abdominal.

IV. Una vez pasadas las agujas se procederá al intercambio de los mangos (Figura 2).

- Control endoscópico.

Con las agujas pasadas, se realiza control endoscópico para confirmar la integridad vesicouretral. En caso de perforación, se retirara/n y recolocará la/s aguja/s por fuera y mas exterior del recorrido inicial, manteniendo el cistoscopio en posición.

- Enhebrado y transferencia de las columnas (Figura 3). Los extremos de las agujas tienen forma de gancho

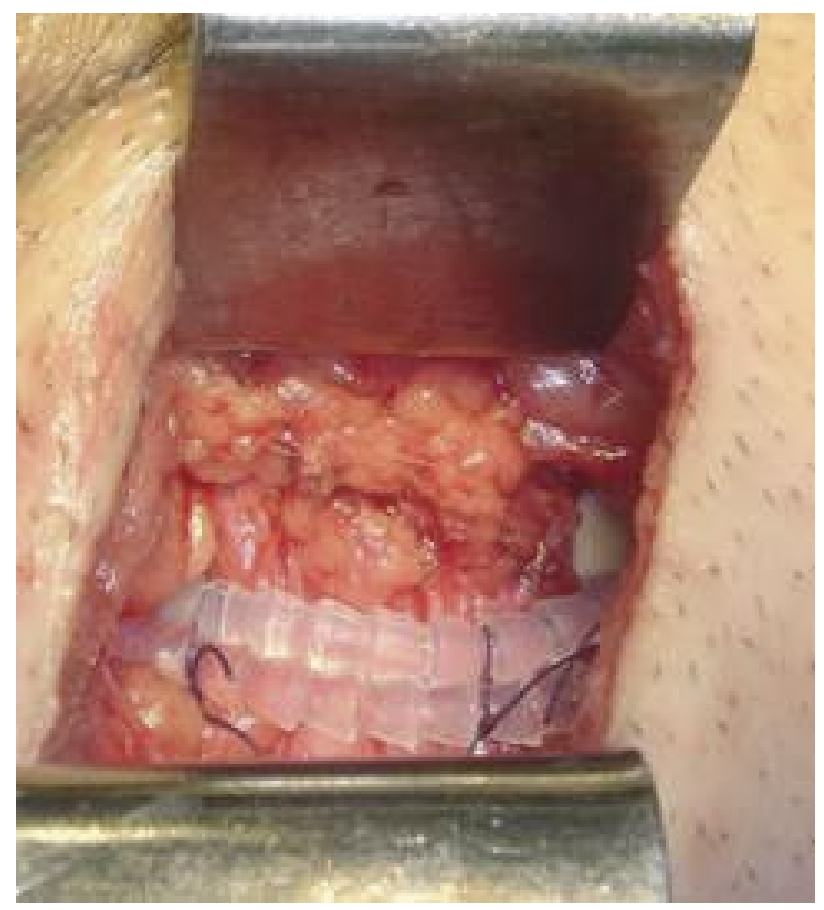

FIGURA 5. Columnas en tejido subcutaneo. 
y permiten enhebrar las columnas del cabestrillo y su paso desde la región perineal a la suprapúbica, labrándose con el arrastre un canal que alojará las columnas en su interior.

- Colocación de las arandelas.

Se colocan las arandelas en una pinza de mosquito se transfieren a las columnas del cabestrillo (Figura 4). El descenso y colocación de las arandelas a través de las columnas se llevará a cabo con el posicionador, este nos permite empujar dentro del tejido celular subcutaneo y colocar las arandelas sobre la aponeurosis.

- Ajuste del cabestrillo.

El ajuste (ascenso y descenso) de las arandelas con el posicionador deberá ser controlado con el cistoscopio y una columna de agua, verificando el cierre de la luz de la uretra al tensionar el cabestrillo. La altura del frasco de suero durante la endoscopía debe ser de $45 \mathrm{~cm}$ de agua, medida desde el pubis del paciente hasta el nivel de agua del frasco. En esta posición la presión uretral retrógrada - PUR será de $45 \mathrm{~cm}$ de la columna de agua.
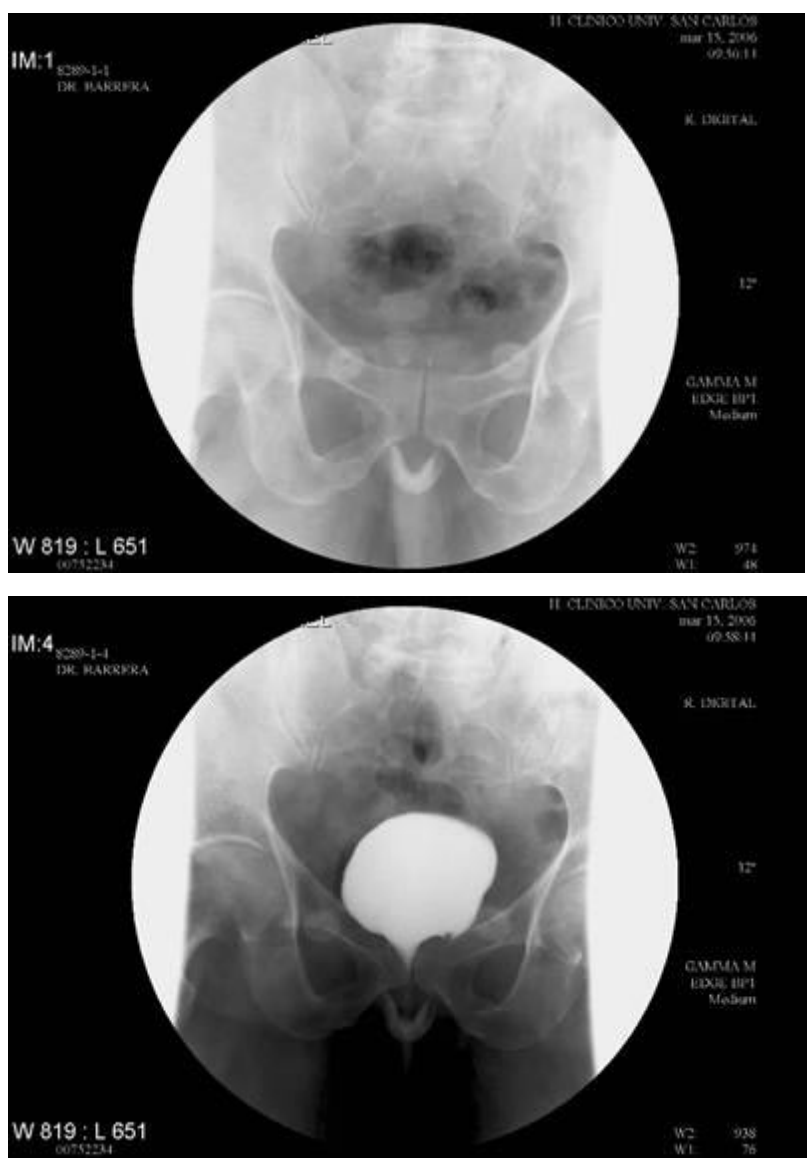

FIGURA 6. Radiografía simple y cistografía en un paciente con Sistema Argus a los 4 meses del implante.
A través de las arandelas se realizará el ajuste del cabestrillo hasta que se detenga el goteo de la línea de infusión. Es importante mantener el centrado de la almohadilla lo cual se consigue fijando una de las aletas del sistema almohadillado en el medio de la uretra con un clamp hemostático atraumático cuidando de no lesionar ni perforar la superficie del sistema.

- Recortar las columnas aproximadamente 6 conos por encima de la arandela. Este exceso de las columnas se debe ubicar en un túnel subcutáneo lo más profundo posible alejándolo de la piel (Figura 5).

- Revisión de la hemostasia.

- Lavado e irrigación con solución antibiótica de la prótesis colocada.

- Cierre de las incisiones abdominal y perineal, evitando dejar drenajes.

\section{4.- Cuidados post-quirúrgicos.}

- Antibioticoterapia. Se mantendrá durante 7 días en total (parenteral y oral).

- Uso de analgesicos y anti-inflamatorios según pauta y necesidades del paciente.

- Retirada de sonda uretral a las 24 horas de la intervención, manteniendose 7 días en caso de perforación.

- Realizar radiografía simple centrada en pelvis para comprobar la adecuada colocación del sling (Figura 6).

- En caso de necesidad se realizará ajuste o desajuste post-quirúrgico. Se recomienda realizar esta operación dentro de los 30 días siguientes a la intervención.

\section{DISCUSIÓN}

El problema de la incontinencia urinaria masculina de esfuerzo está aún por resolver y hasta hace, relativamente, poco tiempo incluso no se le prestaba atención, a pesar de la merma importante en la calidad de vida de los pacientes que padecían este problema. El estandar de oro en el manejo y tratamiento de estos pacientes ha sido e incluso sigue siendo el esfínter urinario artificial $(5,8)$, aunque si bien es cierto esperamos que esto sea así por poco tiempo pues se trata de un artilugio que trata la incontinencia urinaria de forma poco fisiológica, ya que comprime toda la uretra y puede desarrollar atrofia y erosion y además requiere un adiestramiento especializado por parte del paciente. Sea como fuere produce una importante morbi-mortalidad $(9,10)$. 
Las diferentes alternativas terapéuticas en el tratamiento de la incontinencia urinaria de esfuerzo después de cirugía prostática (9-12), deberán adecuarse a las necesidades de cada paciente y aún no tenemos la solución ideal. En este sentido debemos mencionar como alternativas al esfínter urinario artificial otros diferentes sistemas.

Algunos de estos sistemas pretenden colapsar la uretra con su aplicación (inyecciones y balones periuretrales) $(12,13)$ y otros aumentar la presión uretral de cierre en Valsalva mediante operaciones de cabestrillo sobre la uretra bulbar por fijación de la malla a la aponeurosis de los rectos mediante el paso de agujas (sistemas de tensión regulable y sistemas autorregulables) $(1,2,7,11,14)$ o mediante anclaje de la malla con tornillos de titanio a hueso (sin mecanismo de regulación). La principal complicación de estos sistemas de cabestrillo es el dolor perineal o genital (9).

¿Qué sistema será el mejor? Es una pregunta de difícil respuesta y el ajuste para lograr la compresión sin obstrucción es un desafio técnico (15). Lo cierto es que la utilización de cabestrillos se torna como uno de los ideales, ya que permite la regulación y no comprime toda la circunferencia de la uretra. El cabestrillo descrito por Schäeffer ha sido de utilidad incluso después de radioterapia adyuvante (1) Se implanta de una forma sencilla y reproducible y puede ser considerado un sistema con una adecuada relación coste-beneficio, por lo que pronto podremos contrastar resultados con otros centros e incluso realizar estudios multicentricos que permitirán obtener mejores conclusiones.

\section{CONCLUSIONES}

1. Se trata de un sistema seguro, fácil de implantar, reproducible, con pocas complicaciones y una buena relación coste beneficio.

2. Sus resultados son comparables al estandar de oro, pero con las siguientes ventajas: inmediata recuperación del control miccional y no requiere entrenamiento por parte del paciente.

3. Este trabajo no pretende aportar nuestra corta experiencia, que simplemente incluye 5 pacientes, aunque si queremos mencionar que se encuentran en la actualidad continentes y con una buena calidad de vida.

4. Nuestro objetivo será publicar nuestros resultados, cuando podamos aportar un seguimiento mínimo.

\section{BIBLIOGRAFÍA y LECTURAS} RECOMENDADAS ( ${ }^{*}$ lectura de interés $y^{* *}$ lectura fundamental)

**1. ROMANO, S. V.; METREBIAN, S.E.; VAZ, F. y cols.: "An adjustable male sling for treating urinary incontinente alter prostatectomy: a phase III multicentre trial". BJU Int.; 97: 533. 2006.

**2. SCHAEFFER, A.J.; CLEMENS, J.; FERRARI, M. y cols.: "The male bulbourethral sling procedure for post-radical prostatectomy incontinence". J. Urol.; 159: 1510. 1998.

*3. STERN, J.; CLAMEN, J.; TIPLITSKY, S. y cols.: "A. Long-term results of the bulbouretral sling procedures". J. Urol.; 173: 1654. 2005.

*4. CLEMENS, J.; SCHAEFFER, A.J.; BUSHMAN, W.: "Bulbourethral sling procedure". Contemporary Urology. 68. 2002.

*5. SCHAEFFER, A.J.: "Editorial: Prostatectomy incontinence". J. Urol.; 167:602. 2002.

*6. COMITER, C.V.; SULLIVAN, M.P. y YALLA, S.V.: "Retrograde leak point pressure for evaluating post-radical prostatectomy incontinence". Urology; 49:231. 1997.

*7. SOUSA, A.; RODRIGUEZ, J.; URIBARI, C. y cols.: "Externally readjustable sling for the treatment of male stress urinary incontinente: points of thecnique and preliminary results". J. Endourol.; 18: 113. 2004.

*8. SANCHEZ-ORTIZ, R.F.; BRODERICK, G.A.; CHAIKIN, D.C. y cols.: "Collagen injection therapy for post-radical retropubic prostatectomy incontinence: role of Valsalva leak point pressure". J. Urol.; 158:2132. 1997.

*9. HAAB, F.; TROCKAMAN, B.; ZIMMERN, P. y cols.: "Quality of life and continence assessment ot the artificial urinary sphincter in men with minimum 3'5 year of followup". J. Urol; 158: 435439. 1997.

*10. CERQUEIRA, M.; XAMBRE, L.; SILVA, V. y cols.: "Sling bulbouretral. Experiencia del Servicio”. Act. Urol. Esp.; 29:401. 2005.

*11. PETROU, S.P.: "Treatment of prostatectomy incontinence: is the bulbourethral sling a viable alternative to artificial urinary sphincter?". J. Urol. 2003; 170: 1044-1045.

*12. MacDIARMID, S.A.: "Incontinence after radical prostatectomy: pathophysology and management". Curr. Urol. Rep.; 3: 209. 2001.

*13. KRANE, R.: "Urinary incontinence after treatment for localized prostate cancer". Mol. Urol.; 4: 279. 2000.

*14. MIGLIARI, R.; PISTONELI, D.; DE ANGELIS, M.: "Polypropilene sling of the bulbar uretra for post-radical prostatectomy incontinente". Eur. Urol.; 43: 152. 2003.

*15. CLEMENS, J.; BUSHMAN, W. y SCHÄEFFER, A.: "Urodynamic analysis of the bulbourethral sling procedure". J. Urol.; 162: 1981. 1999. 